In response to a request from the SSE, health counselling and health guidance through visits were provided, but those services were not actively used among SSEs.

Conclusions Observed differences between ROHC and WHC were considered attributable to the presence or absence of industry physicians and authority of occupational health nurses. In Japan, it is important to strengthen the authority of occupational public health nurses, and to consider ways of addressing the occupational health needs in SSEs.

\section{TRUCK DRIVERS' HEALTH MANAGEMENT IN JAPAN BY DRIVING OPERATIONS MANAGERS: THEIR EFFORTS AND DIFFICULTIES}

Satoko Shimamoto*, Noriko Nishikido. Nursing School of Health Sciences, Tokai University, Kanagawa, Japan

\subsection{6/oemed-2018-ICOHabstracts. 1416}

Introduction In Japan, the vast majority of transport companies are small or medium-sized enterprises (SME), and do not have any personnel specialising in health management. This report aims to elucidate driving operations managers (DOMs)' efforts as well as difficulties faced therein in order to grasp the current state of and issues in the health management of truck drivers.

Methods We asked DOMs of six SME and three large transport enterprises (LE) questions, related to considerations given to the health of drivers, in semi-structured interviews for each. We first identified efforts being made and difficulties faced in these two groups. Then, we used a content analysis method and analysed them.

Result Regarding efforts being made for the health of drivers, DOMs at SEMs spoke a considerable amount about handling drivers on an individual basis: understanding their usual conditions and discovering their changes through meetings and conversations, as well as checking and listening in a way that takes into account drivers' individual characteristics and backgrounds. DOMs at LE considerably discussed systematic efforts. These included preventions to not increase drivers' burdens and loads, utilising health management professionals. With regard to difficulties, DOMs at SME mentioned the difficulty of interpreting health examination results as well as that in addressing health issues due to vague judgment criteria.

Discussion Differences in the approaches of LE and SME became clear. At LE, health management was being carried out systematically in coordination with health management professionals as part of driving operations management. On the other hand, at SME, drivers' health was attended to on a more case-by-case basis by. It appears that there is a need for DOMs at SME to have more enthusiasm and competence when it comes to health management, as well as to receive more supports in, for example, interpreting health information.

\section{AUVA APPS FOR IMPROVED HEALTH AND SAFETY}

Karin Sturm. Austrian Workers' Compensation Board, Vienna, Austria

\subsection{6/oemed-2018-ICOHabstracts. 1417}

The new generations of smartphones and tablets are increasingly being used for a wide variety of purposes. The Austrian Worker's Compensation Board wants this trend to continue and has begun developing a number of different apps following extensive preparatory work.

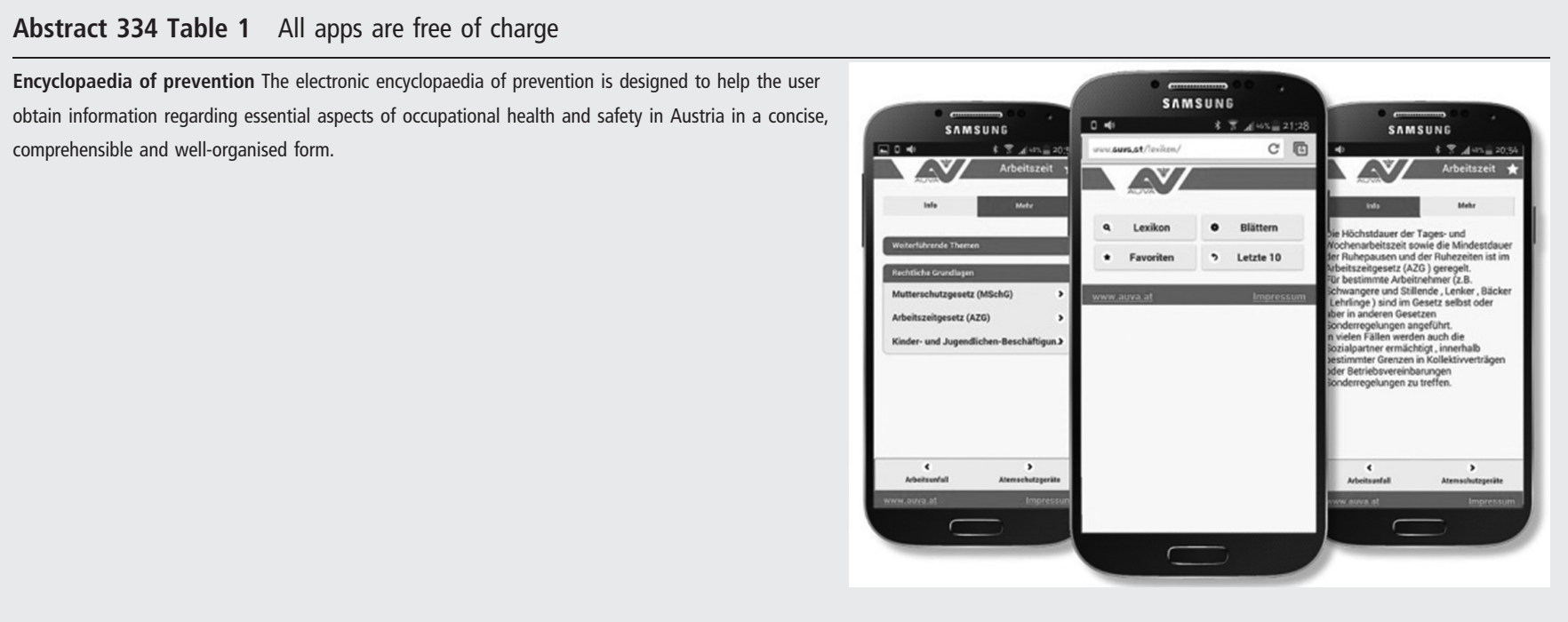


Health and safety management

The 'health and safety management' app was designed as an app to support the gradual introduction to the company's own health and safety management, for assessing the completeness of health and safety management, for carrying out internal audits and for carrying out health and safety inspections using standardised questionaries'.

\section{First Aid - Hand Injuries}

The app provides an overview of the different types of hand injuries. The user obtains important information regarding what to do in the event of emergency. Illustrations accompany you step-by-step through the first aid procedure.

\section{Computer workstation}

The tablet app 'Computer workstation' is based on the field of ergonomics. It is aimed at anybody who works at a computer workstation. The app offers support in setting up this computer workstation in the most ergonomic way possible and provides assistance in detecting any shortcomings.
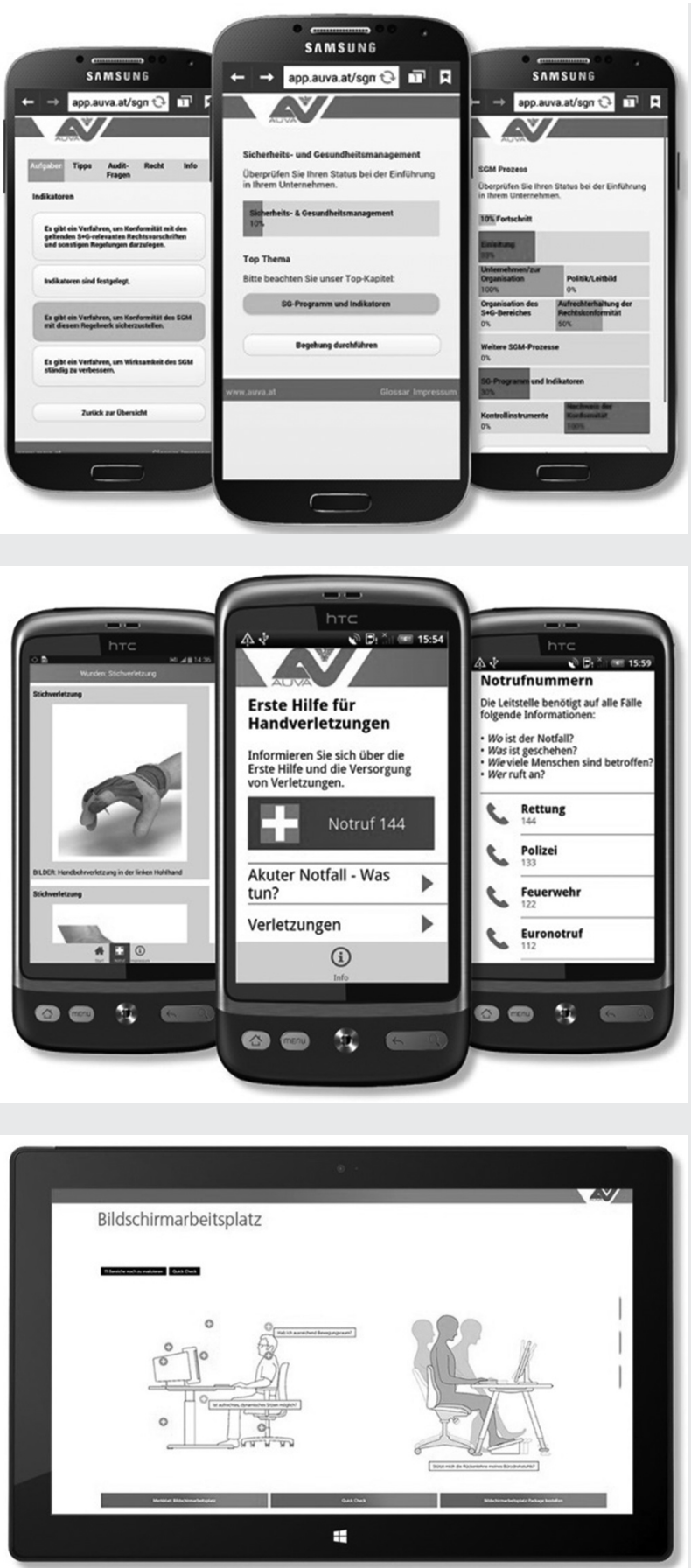